\title{
Laparoscopic Management of Rectus Sheath Hematomas
}

\author{
Dina Chamsy, MD, Deirdre Lum, MD, Suketu Mansuria, MD \\ Center for Minimally Invasive Gynecologic Surgery, Department of Obstetrics, Gynecology and Reproductive Sciences, \\ Magee-Womens Hospital of UPMC, Pittsburgh, PA, USA (all authors).
}

\begin{abstract}
Introduction: Rectus sheath hematomas can result from shearing of rectus muscle fibers or injury to the inferior epigastric vessels, which is the most common vascular injury to occur at the time of laparoscopic surgery.

Case Description: We describe a case of a rectus sheath hematoma that was diagnosed 5 days after operative laparoscopy. We review the clinical presentation, diagnostic criteria, and therapeutic options of managing rectus sheath hematomas, and we describe the innovative use of laparoscopy for hematoma drainage after failed conservative management.
\end{abstract}

Discussion: Rectus sheath hematomas that fail conservative management can be effectively drained laparoscopically. This minimally invasive approach provides rapid symptom relief and patient recovery.

Key Words: Hematoma, Surgical instruments, Complication, Laparoscopy.

Citation Chamsy D, Lum D, Mansuria S. Laparoscopic management of rectus sheath hematomas. CRSLS e2014.00011. DOI: 10.4293/CRSLS.2014.00011.

Copyright (C) 2014 SLS This is an open-access article distributed under the terms of the Creative Commons Attribution-Noncommercial-ShareAlike 3.0 Unported license, which permits unrestricted noncommercial use, distribution, and reproduction in any medium, provided the original author and source are credited.

Disclosure of conflicts of interest and sources of financial support: Drs. Chamsy and Lum have nothing to disclose. Dr. Mansuria serves as a consultant for Olympus and has helped with product development and given presentations on surgical techniques for this company.

Address correspondence to: Dina Chamsy, MD, Magee-Womens Hospital of UPMC, 300 Halket St, Pittsburgh, PA 15213, USA. Telephone: (412) 641-1440, Fax: (412) 641-1133, E-mail: chamsydj@upmc.edu

\section{INTRODUCTION}

Rectus sheath hematomas are usually the result of an inferior epigastric vessel injury or shearing of rectus muscle fibers, which can occur at the time of accessory trocar placement. Most stable rectus sheath hematomas are managed expectantly or treated with ultrasonography or computed tomography (CT)-guided drainage. To our knowledge, this is the first case report to discuss a laparoscopic approach to drain a rectus sheath hematoma diagnosed in the postoperative period.

\section{Case}

Our patient is a 34-year-old gravida 2 para 2 woman who underwent a total laparoscopic hysterectomy for management of pelvic pain and menorrhagia. A peritoneal entry was achieved through an open-entry technique. Three 5-mm accessory ports were placed in the bilateral lower quadrants and suprapubically after laparoscopic identification of the inferior epigastric vessels and bladder to avoid injury. The hysterectomy was uncomplicated with an estimated blood loss of $100 \mathrm{~mL}$. At the end of the procedure, the accessory ports were removed under direct laparoscopic visualization and the sites were hemostatic. Postoperatively, the patient had an acute drop in hemoglobin level from 13.4 to $8.5 \mathrm{~g} / \mathrm{dL}$. The hemoglobin level was measured again and was unchanged. The patient was asymptomatic and was discharged home on the first postoperative day.

On postoperative day 5 , the patient presented to the emergency department with orthostatic dizziness and sharp right lower quadrant pain. She was febrile, with a temperature of $38.3^{\circ} \mathrm{C}$, and tachycardic, with a heart rate of 110 beats per minute. Physical examination showed a tender $5 \times 5-\mathrm{cm}$ abdominal mass, medial to the right lower quadrant accessory trocar site. The skin incisions were intact with no purulent drainage; therefore wound cultures were not taken. Blood cultures were drawn and were negative on two occasions. Laboratory testing showed no leukocytosis but a further drop in hemoglobin 
level to $7.2 \mathrm{~g} / \mathrm{dL}$. CT imaging of the abdomen and pelvis with intravenous contrast showed a $10.6 \times 8.3-\mathrm{cm}$ right lateral abdominal wall hematoma with no evidence of active extravasation. The mass appeared to be subfascial yet confined to the preperitoneal space (Figure 1).

We admitted the patient with a presumptive diagnosis of an infected rectus sheath hematoma. We opted for conservative management including pain control and intravenous antibiotics. We also transfused with $1 \mathrm{U}$ of packed red blood cells for management of symptomatic anemia in the patient. The interventional radiology department was consulted regarding possible drainage of the infected hematoma. They deferred immediate drainage and would proceed only if conservative management failed. Defervescence occurred, and the patient remained afebrile for $>48$ hours. Her pain overall improved, and there was no evidence of wound infection or peritoneal signs. She was discharged home on hospital day 4 with a plan to complete a 14-day course of oral antibiotics.

The patient presented to the emergency department 2 days later with recurrence of a low-grade fever $\left(37.9^{\circ} \mathrm{C}\right)$ and worsening right lower quadrant abdominal pain. Her hemoglobin level was stable at $10.0 \mathrm{~g} / \mathrm{dL}$. The hematoma size and appearance were unchanged on a repeat CT scan. Because of failed conservative management, we decided to proceed with laparoscopic drainage of the hematoma. Given that the hematoma appeared subfascial and preperitoneal on imaging, we believed that it would be easily accessible laparoscopically.

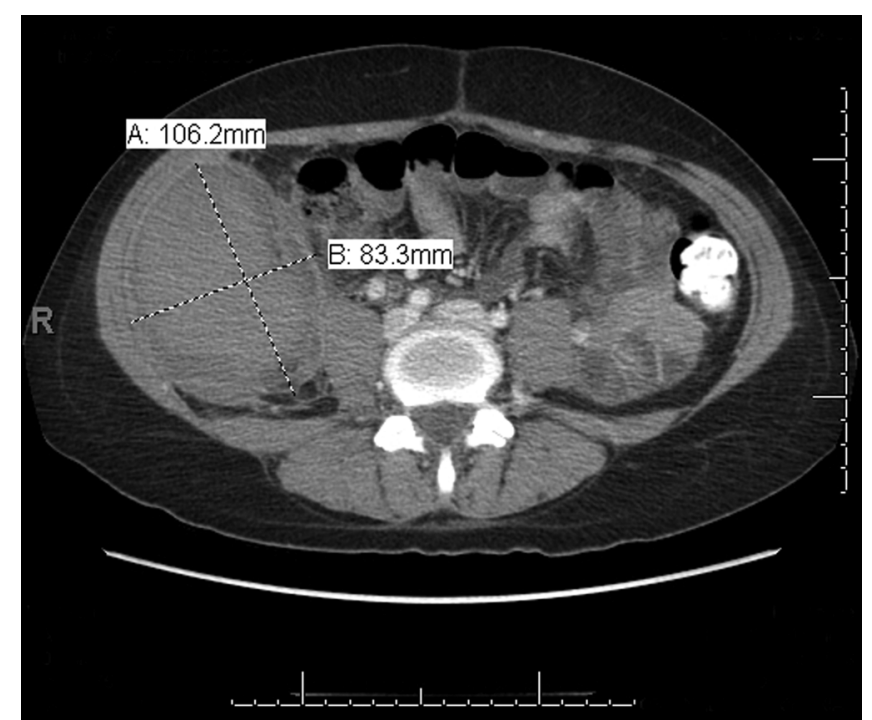

Figure 1. CT scan transverse section of abdomen shows right $10.6 \times 8.3-\mathrm{cm}$ rectus sheath preperitoneal hematoma.
Intraoperatively, we opened the right lower quadrant skin incision but could not express any blood, confirming that the hematoma was subfascial as seen on the CT scan. On laparoscopy, we were able to visualize a large right-sided rectus sheath hematoma, bulging into the peritoneal cavity (Figure 2). The defect in the peritoneum from the previous right lower quadrant port site was visible, and there was no active bleeding from this site. We introduced the suction irrigator tip through the peritoneal defect and aspirated $250 \mathrm{~mL}$ of viscous blood. We extended the peritoneal defect superiorly and inferiorly to approximately $6 \mathrm{~cm}$ (Figure 3). No active bleeding was observed. A small amount of clot was noted near the right external iliac vessels. To avoid inadvertent injury to these major blood vessels, we proceeded with copious irrigation of the hematoma cavity with $3 \mathrm{~L}$ of normal saline solution.

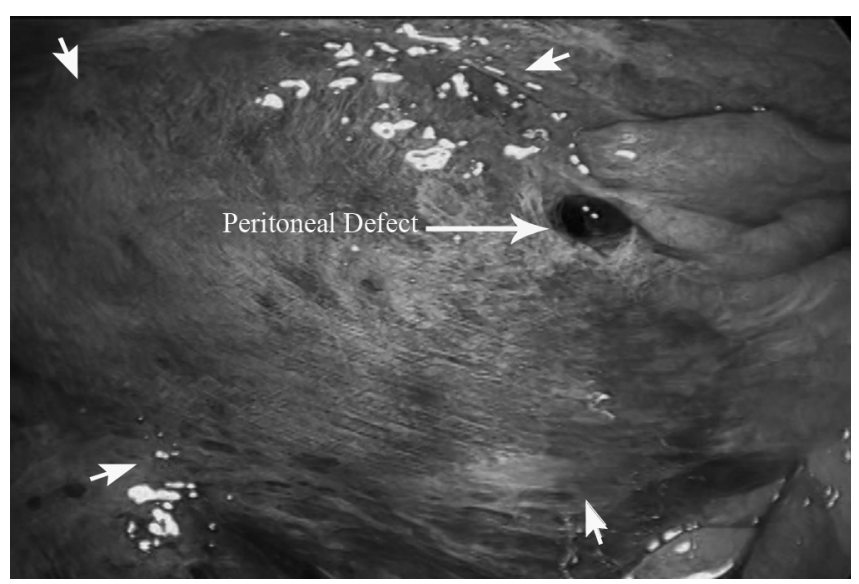

Figure 2. Laparoscopic image shows right rectus sheath hematoma (arrowheads) and peritoneal defect from previous accessory port site.

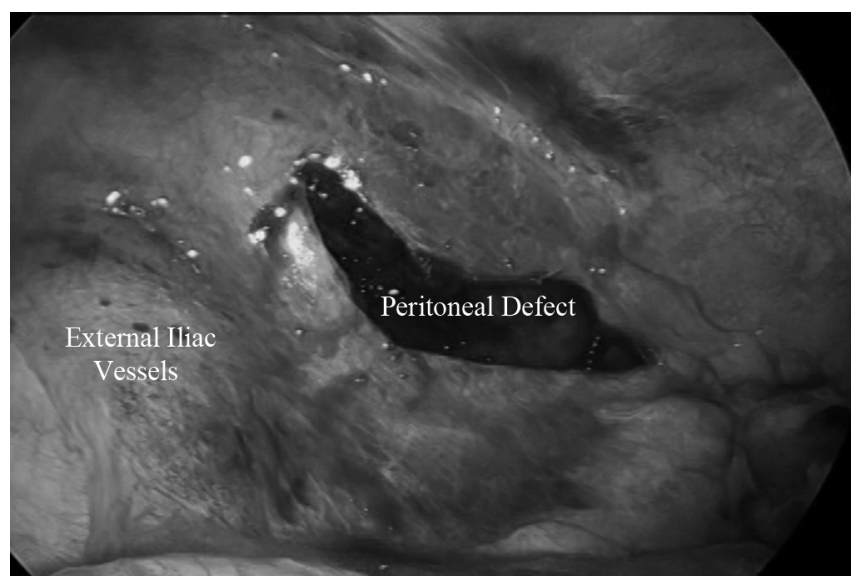

Figure 3. Laparoscopic image shows right rectus sheath hematoma after extension of peritoneal defect to enhance exposure. 
We then placed a Jackson-Pratt drain through the right lower quadrant port and positioned it within the hematoma cavity to allow drainage of liquefied blood clots. Postoperatively, the patient had immediate pain relief. She remained afebrile, and her hemoglobin level was stable. She was discharged home on postoperative day 2 in stable condition. The Jackson-Pratt drain had minimal output and was removed before the patient was discharged. At her postoperative visit, the patient was doing well. Her recovery time from the hysterectomy was not affected by the laparoscopic hematoma drainage, and she was able to return to work as scheduled.

\section{Discussion}

Rectus sheath hematomas usually result from injury to the inferior epigastric artery or vein or shearing of rectus muscle fibers. They are commonly iatrogenic after paracentesis or laparoscopic surgery. The exact incidence of rectus sheath hematoma at the time of trocar insertion is not known; a retrospective study reviewing 15,422 laparoscopic surgeries in Japan reported a rate of $0.46 \%{ }^{1}$

Injury to the inferior epigastric artery is typically diagnosed in an acute setting, either intraoperatively or in the immediate postoperative period, as patients rapidly show signs of hemodynamic compromise. Acute bleeding from the inferior epigastric vessels noted at the time of surgery can be controlled by introducing a laparoscopic bipolar sealing device through the contralateral trocar port and coagulating the bleeding vessels. If the vessels are completely transected, they often retract superiorly and inferiorly. In this case the surgeon must coagulate both transected ends of the vessels. Another technique consists of applying two figure-of-eight sutures on either side of the trocar site, laparoscopically or transabdominally. If the transabdominal approach is used, knots should be tied inside the trocar incision to avoid skin strangulation. The skin incision may need to be extended in this situation. Transabdominal suture ligation can be facilitated with the use of a needlepoint fascial closure device. ${ }^{2}$

Our patient was diagnosed with a rectus sheath hematoma 5 days postoperatively, indicating that the hematoma was likely caused by tearing of muscle fibers and small perforating blood vessels, which did not result in brisk bleeding leading to hemodynamic compromise. In fact, at the time of her laparoscopic hematoma drainage on postoperative day 10, the right inferior epigastric vessels were visualized and appeared intact, medial to the site of the hematoma. In addition to signs of hemodynamic compromise, patients with rectus sheath hematomas typically present with localized abdominal pain and a palpable mass on physical examination with occasional overlying ecchymoses. Peritoneal signs, mimicking an acute intraabdominal process, may be present on physical examination because the hematoma can extravasate intraperitoneally and cause peritoneal irritation. Our patient had the typical clinical features of localized abdominal pain and a palpable mass on physical examination. In addition, a fever developed, indicative of an infected rectus sheath hematoma.

Diagnosis of rectus sheath hematomas can be confirmed by CT scan imaging. A retrospective study published in 1996 reviewed the appearance of 13 rectus sheath hematomas on CT scan imaging over a period of 4 years. ${ }^{3}$ The authors proposed a radiologic classification to aid in a therapeutic approach to rectus sheath hematomas. They described 3 types of hematoma, based on the location within the abdominal wall. Type I hematomas are intramuscular and therefore always unilateral. Type II hematomas accumulate between the rectus muscle and the underlying transversalis fascia and can spread to the contralateral side if large enough. Type III hematomas accumulate between the rectus muscle and transversalis fascia but also spread to the preperitoneal space. These can expand inferiorly to the prevesicular space and occasionally cause hemoperitoneum.

In the setting of a rapidly expanding hematoma in a patient who is hemodynamically unstable, emergent vascular embolization or surgical intervention is often necessary. Stable rectus sheath hematomas frequently resolve spontaneously with supportive medical management including pain control and blood transfusion. Type I hematomas do not require hospitalization. Type II hematomas frequently require hospital admission for hemodynamic monitoring and medical management. Type III hematomas often require blood transfusions and a longer recovery period. Our patient had an infected type III hematoma that failed conservative management with intravenous antibiotics and a blood transfusion. We considered possible therapeutic options including percutaneous hematoma drainage by interventional radiology or drainage through a laparoscopic approach. Pulsed ultrasound therapy to accelerate hematoma resorption has been reported but is currently not the standard of care. ${ }^{4}$

The drawback to ultrasonography or CT-guided percutaneous drainage is that it is not readily available in all facilities. Moreover, not all abdominal wall hematomas are amenable to percutaneous drainage. The chance of successful drainage by a percutaneous approach decreases in 
the setting of loculated collections or clotted blood because the drainage catheter is of small caliber and can easily become obstructed. ${ }^{5}$ Consequently, the drain must remain in place for several days, and symptoms are not relieved immediately. In the setting of an infected hematoma, a partially obstructed drain can potentially become a nidus for infection.

A laparoscopic approach is beneficial because it is minimally invasive and provides immediate symptomatic relief. Moreover, it allows direct visualization and inspection of the hematoma site to rule out any active bleeding. Risks, which include the risks related to surgery and anesthesia, are minimal. Recovery time is similar to that of most minimally invasive laparoscopic procedures, and the patient can avoid the inconvenience of prolonged percutaneous catheter care.

Although trocar insertion under direct laparoscopic visualization should prevent injury to the inferior epigastric vessels with subsequent hematoma formation, rectus sheath hematomas can still occur due to shearing of rectus muscle fibers and disruption of small subfascial vessels. This risk can be minimized by avoiding extreme use of force at the time of trocar insertion. Trocars should be inserted in a controlled manner, with a twisting motion to avoid excessive tissue tension. Sharp trocars are preferred because they require less force to penetrate the abdominal wall and thereby reduce the risk of tissue shearing and vascular injury. Establishing adequate peritoneal pressure is also essential; poor peritoneal insufflation increases abdominal wall laxity, which decreases tissue resistance and makes trocar insertion more challenging. ${ }^{6}$

The key to proper placement of lateral accessory trocars is to first identify the inferior epigastric vessels. These vessels course along the ventral aspect of the anterior abdominal wall, just lateral to the medial umbilical ligaments. The medial umbilical ligaments are a key landmark in the identification of the inferior epigastric vessels because they are easily identifiable, even in obese patients. After these vessels are identified, lateral trocars should be placed under direct visualization to ensure that peritoneal entry is lateral to the vessels. Our usual technique is to identify the vessels and make the skin incisions at or lateral to the McBurney point, approximately one-third of the distance between the anterior superior iliac spine and the umbilicus. Trocars are then inserted at an angle perpendicular to the contour of the abdomen, ensuring that they penetrate lateral to the rectus sheath and through the aponeurosis of the external and internal oblique muscles, thereby avoiding trauma to the rectus muscle fibers and inferior epigastric vessels. After trocar removal, trocar sites should be carefully inspected laparoscopically after reducing pneumoperitoneum to unmask potential bleeding. Postoperatively, physicians should have a low threshold to investigate any sign of hemodynamic compromise or unexpected drop in hemoglobin level.

In conclusion, although most laparoscopically induced rectus sheath hematomas can be prevented with the use of proper surgical technique, all surgeons should be prepared to manage this relatively common complication. Surgeons should have a low threshold to suspect a rectus sheath hematoma when there is any sign of hemodynamic compromise after laparoscopic surgery or if a patient expresses worsening localized abdominal pain with a palpable mass on physical examination. If conservative management fails, laparoscopic drainage of the rectus sheath hematoma is a feasible minimally invasive approach that provides rapid symptom relief and patient recovery.

\section{References:}

1. Hashizume M, Sugimachi K. Needle and trocar injury during laparoscopic surgery in Japan. Surg Endosc. 1997;11:1198-1201.

2. Nezhat C, Nezhat F, Nezhat C. Laparoscopic Access. In: Nezhat's Operative Gynecologic Laparoscopy and Hysteroscopy. 3rd ed. New York, USA: Cambridge University Press; 2008: $58-60$.

3. Berná JD, Garcia-Medina V, Guirao J, et al. Rectus sheath hematoma: diagnostic classification by CT. Abdom Imaging. 1996;21(1):62-64.

4. Berná-Serna JD, Sánchez-Garre J, Madrigal M, et al. Ultrasound therapy in rectus sheath hematoma. Phys Ther. 2005; 85(4):352-357.

5. Wallace MJ, Chin KW, Fletcher TB, et al. Quality improvement guidelines for percutaneous drainage/aspiration of abscess and fluid collections. J Vasc Interv Radiol. 2010;21:431-435.

6. Bhoyrul S, Vierra MA, Nezhat CR, et al. Trocar injuries in laparoscopic surgery. Am Coll Surg. 2001;192(6):677-683. 